\title{
An Integrated Information System for Route Inspection in Manufacturing
}

\author{
Zhang Qing ${ }^{1}, X_{u}$ Guanghua ${ }^{2}$, Wang Jing ${ }^{2}$, and Liu Dan ${ }^{1}$ \\ 1 Department of Instrument Science and Technology, School of Mechanical \\ Engineering, Xi'an Jiaotong University, Xi'an 710049, P.R. China. \\ aii@mail.xjtu.edu.cn. \\ 2 State Key Laboratory for Manufacturing Systems Engineering, Xi'an \\ Jiaotong University, Xi'an 710049, P.R. China. \\ xugh@mail.xjtu.edu.cn.
}

\begin{abstract}
Medium and small equipments in manufacturing are numerous, various and dispersive, so route inspection is the best method to acquire their condition information for its flexibility and low costs. In this paper, an integrated information system for route inspection is constructed to achieve distributed information acquisition on a large scale and centralized information management in a well order. Firstly, the information transfer model of route inspection is erected to describe information demands of route inspection participant. Then, several key problems, such as the instrument for information acquiring, information administration mode, interaction mode and security, are resolved respectively. Finally, three units, workstation, server and client are developed to acquire, manage and utilize the information. They are integrated into a whole system called MTREE, which has been applied into several steel enterprises successfully.
\end{abstract}

\section{Introduction}

Due to the crucial effect of equipment running condition to industry manufacture and profit, computer has been widely used to manage the equipment condition information. Many systems are developed to enhance the efficiency of equipment management and have achieved plentiful success application. But the condition information is not isolated, it contacts with other information closely, such as maintenance plan, spare part storage, production schedule etc. So the integration of condition management becomes a new research issue. In 1999, the ARC Advisory Group originated the terminology "Plant Asset Management" (PAM) [1] to describe systems specifically intended to enable the full scope of asset management function in a plant. The primary elements of such a system include condition monitoring,

Please use the following format when citing this chapter:

Qing, Z., Guanghua, X., Jing, W., Dan, L., 2006, in International Federation for Information Processing, Volume 205, Research and Practical Issues of Enterprise Information Systems, eds. Tjoa, A.M., Xu, L., Chaudhry, S., (Boston:Springer), pp.479-487. 
maintenance management, and reliability management modules with integration to one another and to further support the enterprise resource plan (ERP). A framework for intelligent e-maintenance system [2], which provides web-enabled maintenance service by equipment manufacturer, is presented. Depending on the integration of information system, customers i.e. the equipment users can pay their attention only to making the production. Such new ideas are put forward in succession, but most of them are not realized yet because of some unresolved problems, foremost of which is the overall acquisition of condition data.

The condition management is the basis of whole equipment information management. Its function includes acquiring the condition data and utilizing the data effectively. According to the acquisition mode, the condition management system is classified as online, portable and route inspection, and applied in difference instance [3]. The online system monitors the key equipment, the portable system is used to analyze the fault broken out, and the route inspection system, which is implemented by inspector's periodic data acquiring, is adapted to for the dispersive medium and small equipments. For mastering the multiform equipment condition roundly, these three modes must cooperate closely. However, due to the shortage of technique and platform support, the development of route inspection is slower than the online and portable. It is an obstacle to establish the integrated condition management system for enterprise.

In this paper, a whole system, which consists of three units, workstation, server and client, is established to resolve the management problem of route inspection information in manufacturing. The content of the paper is laid out in 6 sections with this section as the introduction. The characters of condition information acquired by route inspection are investigated in Section 2. Section 3 erects the information transfer model, which analyzes the information demands of different user. Section 4 and 5 describe the system design in some emphases and the system construction respectively. Finally, the application instance is introduced in Section 6.

\section{The characters of condition information acquired by route inspection}

There are a large number of the medium and small equipments in the industry enterprise. The online and portable systems can't inspect them continuously due to the expensive costs. So route inspection is the best data acquisition method for them. Moreover, the condition information of the medium and small equipments is various. Some are measured by special instrument, some are read from the meters on the equipments and others are observed and recorded by inspectors. Therefore, it is difficult to acquire the information on a large scale. At present, only the important data is collected, and other secondary information is ignored. When the technician and manager judge the equipment condition, a new task has to be arranged to acquire more detailed information over again. On occasion, the technician cannot help 
analyzing the condition in worksite. On this account, the efficiency of inspection is low and the safety of equipment running can't be guaranteed.

To actualize the route inspection, the workstations, whose responsibility are acquiring the condition information and dealing with ordinary maintenance affair, are setting in the workshop, and the equipment is allotted to certain inspector. Since everyone pays attention to the own responsibility, condition information is only mastered by collectors. If the others want to obtain correlative information, they must communicate with the inspector by oral or written report. But the expression style of different technical fields has some diversity, sometime it perhaps causes misunderstand. In the other hand, the information can't be transferred timely. Under the circumstance, the condition information acquired by the route inspection is isolated and can't be used in a high level.

As a result of the inspected equipments' spreading out on every work site, the periodic acquisition is executed by workstation dispersedly. This distributed characteristic makes trouble to the enterprise information integration, whose target is managing and sharing the data and service in whole enterprise. At present, there are two faulty management modes. One gives priority to the workstation. The information is only saved in the workstation and the condition management is carried on independently. The superior department only checks the result of workstation. This mode is unfavorable to the global administration, which deals with the harmony of workstation or workshop. On the contrary, the other mode thinks much of the uniform administration. The inspection plan is set and executed uniformly, and the condition information is applied in certain technical department. By this mode, the administration load is centralized and the flexibility of route inspection is lost. Therefore, new management modes should be brought forward to solve this conflict.

\section{The information transfer model of route inspection}

In workshop, methods, such as observing, vibration or noise measuring, oil sample analyzing and whole performance evaluating etc, are utilized to inspect the equipment. So numerous information including appearance, vibration, temperature, metal residua and control parameter are acquired and analyzed to identify equipment condition. The information is classified into three types: measurement information, display information and observation information. The measurement information includes vibration and temperature signal, which are acquired easily and sensitive to the equipment abnormality. The display information includes performance and process parameters, which is displayed by the meters on the equipments. The observation information includes observation results, which describe some appearance characteristics of equipment by some terms, such as clean, dirty, incline, in repair, etc.

The participants of route inspection include data collector, plan administrator, diagnosis technician and equipment manager, and they belong to the workstation, technical office and manage section of workshop or head office respectively. The 
condition information flows from the bottom department to the upper department, and is appended new content continuously by every layer. According to the flow process, a general information transfer model is erected as shown in Fig. 1. In workstation, the plan administrator creates inspection plan and arranges the data collector to acquire three types information periodically. If the condition can't be identified correctly due to the low technique level, the information is transferred to technician office and analyzed by the diagnosis specialist further. The workstation reports equipment abnormality, inspection arrangement and achievement to the workshop. Combining the reports with diagnosis conclusion and repair advice, which is presented by diagnosis technician, the workshop can organize the maintain work, or dispose new inspection task. In this way, the condition management is almost achieved in a basic unit. But the production and equipment maintenance of workshop must obey the global arrangement of enterprise. Therefore, the maintain plan, production plan and spare part plan of the workshop should be confirmed by the head office. It can be found that the condition management to route inspection is a special part of the enterprise information management.

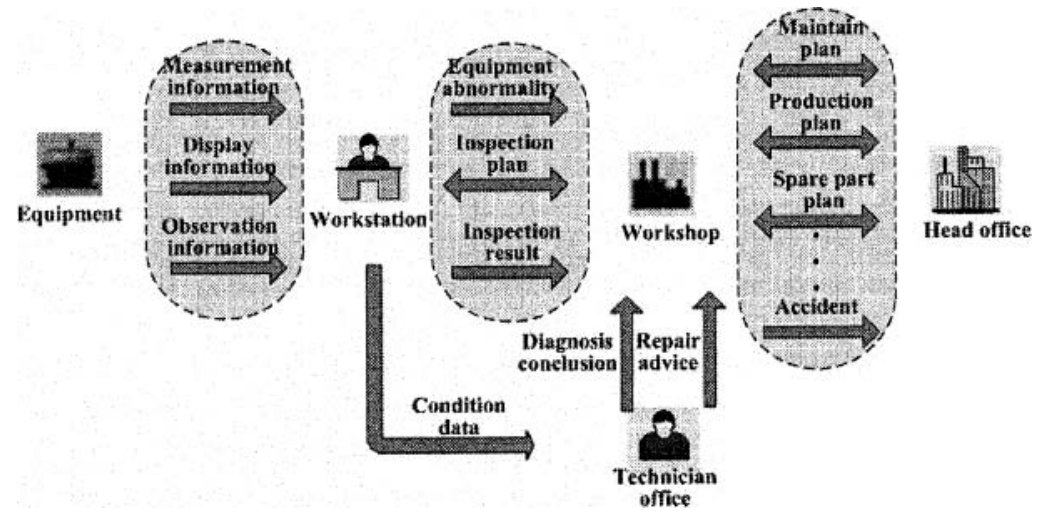

Fig. 1. The information transfer model of route inspection

\section{System design}

The function of route inspection system is to acquire, manage and utilize the numerous and various condition information. In this section, a route inspection system based on intranet, called MTREE, is design and some emphases are described as follows.

1) The instrument for information acquiring. The condition information is classified as measurement information, display information and observation information in the information transfer model, so the corresponding instrument is 
designed to achieve their acquisition. A serial of convenient measurement instrument is developed to acquire the vibration and temperature signal. Because their function emphasizes on the frequent measurement, not the analytic capability, the cost is reduced greatly. It is favorable for route inspection on a large scale. On the other hand, the display information and observation information are acquired by the palm handheld, which replaces the paper and pen with the help of digital production. These two types of instrument, which can communicate with the PC to accept inspection plan and callback the data conveniently, are hardware basis of the system.

2) The information organized according to user role. The participants of route inspection take charge of different work and their information demand is different also. The system provides four subsystems to offer information service. The detailed inspections plan, which denotes the attribute of inspection point such as type, reference data, normal domain and location, is sent to the instrument. The data collectors can accomplish their work depending on the subsystem in instrument. The Route Workstation subsystem provides the function including creating, sending, and receiving and checking inspection plan and analyzing data to enhance the work efficiency of the plan administrator. In addition, two clients, Data Analyzer and Condition Browser, are developed to satisfy the information demand of the diagnosis technician and equipment management. The Data Analyzer provides the abundant diagnosis methods, and the Condition Browser provides the statistical information which help to make decision.

3) Connecting workstation with enterprise. To solve the conflict between the independence of workstation and the information integration of enterprise, two types of database are established. One is the workstation database, which is saved in the workstation computer and supports the Route Workstation subsystem. The other is the enterprise database. Through an automatic running program, the information in all workstation databases is gathered and uploaded to the enterprise database timely. The clients access the enterprise database to get the equipment condition information and input some new information. By this means, the workstation can work independently while the upper department can master the equipment condition.

4) Administration of data acquisition. For ensure the data to be acquired in the required location and time, the electronic button, which has a unique number for automatic identification, is fixed on the equipment. When the data collector touch the button with data acquiring instrument, the registration number is read and corresponding inspection task is displayed. By this way, data acquisition is not only high efficient, but also under the strict control.

5) Diagnoses service to technician. Due to the medium and small equipments are various, the special diagnosis method aiming at the certain equipment type can't always fit other equipments. So besides some typical methods, several kinds of comparing methods are provided, which can compare data acquired at different time or from different equipment freely. By the accumulation within a long period, the abundant historical data of normal, abnormal and fault condition is saved in the database. It is regarded as diagnosis knowledge for the future diagnosis and shared in the enterprise. 
6) Information interaction mode. There are two types of information interaction mode, client/server and browser/server, based on network. The advantage of client/server is high reliability, and the disadvantage is hard to maintain due to the program's being installed in every client. On the contrary, the client/server mode utilized the browser such as IE and Netscape to interact. So the program update is achieved easily, but the reliability is degraded. According to their characteristics, the subsystems for workstation and technician office utilize the client/server mode due to their busy data exchange. The subsystem of Condition Browser, which offers information to the workshop or head office administrator, adopts the browser/server mode because the information mainly flows in a single direction.

7) Data security. The information is saved not only in the workstation database but also in the enterprise database. After new data is acquired and saved into workstation database, they are uploaded to the enterprise database in time and the uploaded records are saved in some table. If the upload cannot accomplish due to the network block, the records can be used to resume the upload process when the network is recovered. By this dual saving mode, distributed and centralized, the data security is enhanced. Even the enterprise database is breakdown, the condition data can be still gotten back from the workstation database.

\section{System construction}

According to the design mentioned above, an integrated information system for route inspection, called MTREE, is developed as shown in Fig. 2. Three units, workstation, server and client are integrated to achieve distributed information acquisition on a large scale and centralized information management in a well order. From three levels of acquisition, management and utilization, the management problem of route inspection information in manufacturing is resolved. 


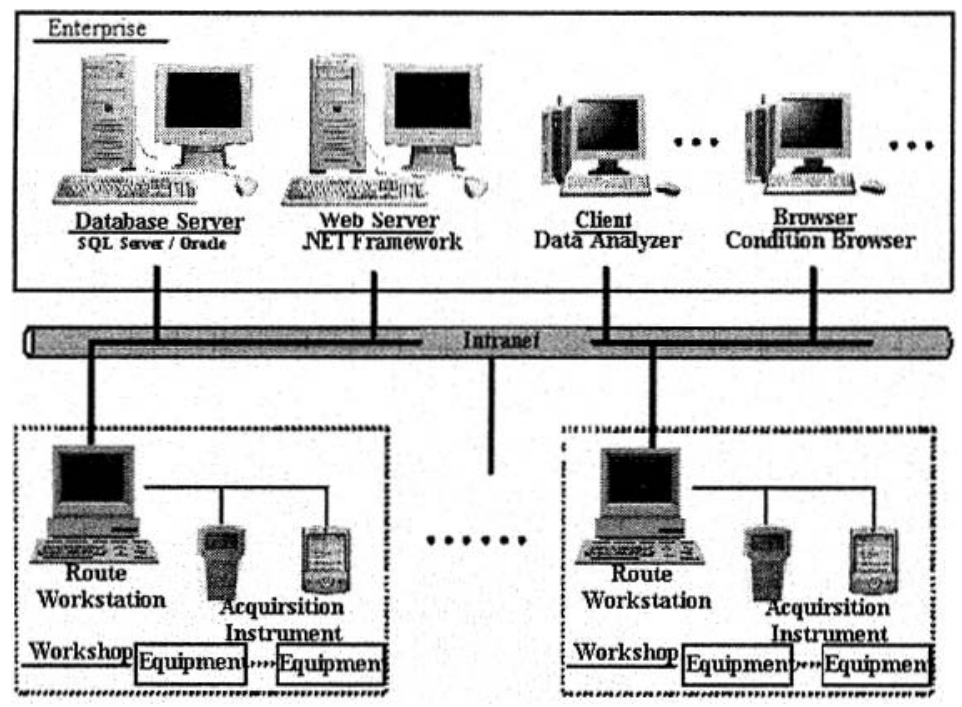

Fig. 2. Contracture of the information management system for route inspection

The Route Workstation subsystem, which works on the desktop database Access, administrates the acquiring work of condition information in workshop. Firstly, the basic information, such as workshop, equipment and inspection point, is inputted. Afterwards, the route plans, which indicate the acquisition task to certain inspection point and in certain period, are created. The plan is sent to the instrument and executed by data collector. Finally, the accomplished plan is callback to the Route Workstation. The plan administrator can deal with the data and make electronic report. In addition, a program is automatic running on the workstation computer to upload the information to the database server.

Servers, including database server and web server, are the management and server center of MTREE. The SQL Sever or Oracle is installed in the database server to save the condition information of the whole enterprise, which includes the condition data and inspection plan uploaded by workstations, diagnosis conclusion and repair advice inputted by technician and the maintenance arrangement and production plan decided by administrator. A maintenance tool is installed in the database sever to backup and restore data and configure the user right. The web server accepts the browser's requests and sends web pages back to the client's computer. Its main function is providing the information service to the administrator.

Data Analyzer and Condition Browser constitute the information utilization platform. The function of Data Analyzer includes some common methods of vibration signal processing, data trend prediction, alarm threshold setting, analysis $\log$ recording and report making, etc. In addition, the representative single data or long-range trend is saved as the case to guide the equipment condition analysis and diagnose. Condition Browser provides the abundant statistic information to the 
administrator, which includes equipment current condition, diagnosis conclusion, repair advice and the actual equipment photo. So the administrator can master the equipment condition and make decision expediently in his office.

\section{Application}

According to the location of database server, the deployment of MTREE can be classified into distributed style or centralized style as shown in Fig. 3. In distributed deployment, each workshop has independent database server, client/browser and route inspection workstation. The superior departments or other workshops can also visit the database server if authorized. In centralized deployment, there is only one database server in the enterprise and all the data are managed concentrated. In this manner, technical service and equipment management is layout in a global angel of view, while the requirement of Data security is enhanced. In spite of the difference of distributed deployment and centralized deployment, information demands of all user rules are the same. So, interfaces and functions of MTREE, which are designed according to user roles, are adapted to different deployment style.
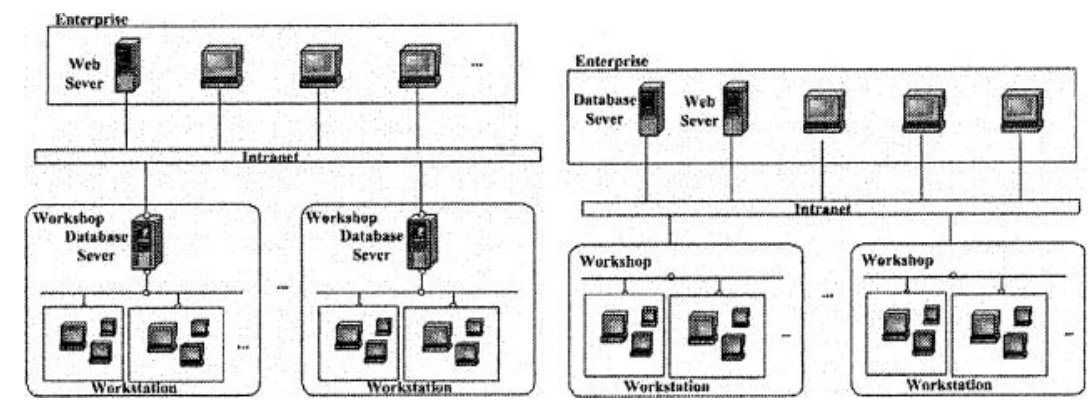

Fig. 3. The deployment of MTREE. The left is distributed and the right is centralized

The integrated information system for route inspection of equipment condition, introduced in this paper, provides a full solution for route inspection management in enterprise, and promotes the development of information management for medium and small equipments. It has been applied in several steel enterprises in P.R. China and improved the efficiency of equipment management significantly. According to the statistic of the WUHAN rolling mill factory, 13 incipient faults are discovered by MTREE in 2003, which decreases a direct loss of over 50 million RMB. 


\section{Acknowledgements}

This work was supported by the National Natural Science Foundation of P.R. China (approval number: 50335030).

\section{References}

1. ARC Advisory Group; http://www.arcweb.com/

2. J. Lee, A Framework for Web-Enabled E-Maintenance Systems, Environmentally Conscious Design And Inverse Manufacturing, In the Proceedings of the EcoDesign 2001, pp. 450-459.

3. M. Xu, Equipment Fault Diagnosis Notebook (Xi'an Jiaotong University Press, Xi'an, China, 1998). 